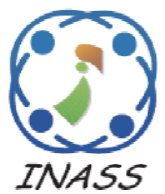

\title{
EEG-Controlled Robot Navigation using Hjorth Parameters and Welch-PSD
}

\author{
M. J. Niegil Francis ${ }^{1 *}$ \\ Mihika Preethi Keran ${ }^{1}$ \\ Rachana Chetan ${ }^{1}$ \\ B. Niranjana Krupa ${ }^{1}$ \\ ${ }^{I}$ Department of Electronics and Communication, PES University, India \\ * Corresponding author's Email: niegilfrancis14@gmail.com
}

\begin{abstract}
This study aimed to find an optimal feature-extraction method for real-time electroencephalography-based navigation. A comparative study of promising methods was done on two-class motor-imagery data. A novel combination of Hjorth parameters and Welch's-power-spectral-density produced the highest two-class accuracy of 0.84 across five subjects. The method is computationally inexpensive and provides short training and execution times that best suit real-time applications. The method yielded an average accuracy of 0.66 on three-class data. The real-time feasibility of the method was tested by implementing an object-retrieving robot and running a navigation experiment. The robot successfully navigated a three-circuit maze using three commands - right, left, and forward - and automatically retrieved the desired object. Along with being ideal for real-time applications, this method utilized only eight electrodes, making it compatible with cheap, portable BCI devices. The method and the application-framework can find commercial utility in the field of healthcare for the elderly and physically-handicapped.
\end{abstract}

Keywords: EEG, BCI, BCI Competition III, Three-class, Welch-PSD, Hjorth parameters, Select-k-Best, SVM, Logistic regression, Robot navigation, Object picking, Path-retracing.

\section{Introduction}

Accidents, strokes, and disabilities leave many individuals immobile or in pain. Their brain signals can be translated into commands to operate robots that can retrieve common household objects for them, thereby assisting their daily lives. This concept can be implemented using a braincomputer interface $(\mathrm{BCI})$, that translates changes in specific energy/

frequency patterns radiated by the user's brain into appropriate commands for robotic systems.

Electroencephalography (EEG) is a common way to record electrical activity generated by the brain in non-invasive systems. Motor imagery (MI) signals are produced when the user imagines moving specific parts of their bodies like their hands or feet. They usually lie in the amplitude range $0.5-100 \mu \mathrm{V}$, and frequency ranges $\mathrm{Mu}$ $(8-12 \mathrm{~Hz})$ and Beta $(13-30 \mathrm{~Hz}) . \mathrm{Mu}$ and Beta rhythms of EEG signals can be used to distinguish between various MI tasks and are therefore analysed in MI based BCI systems.

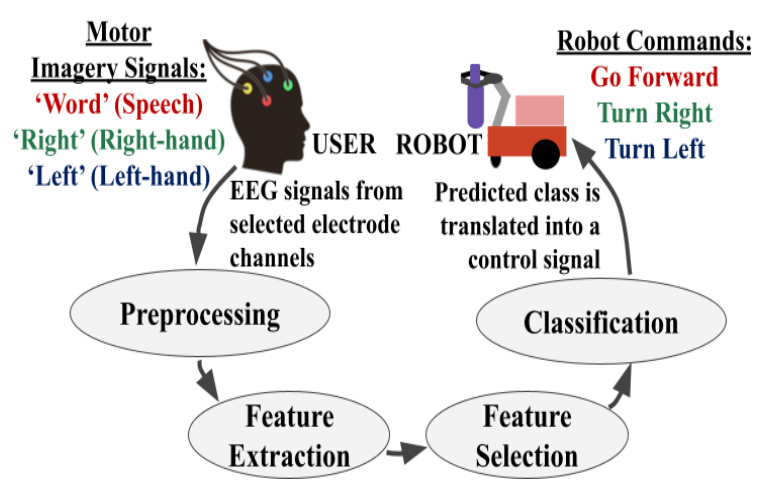

Figure. 1 Flow diagram

In this study, a novel real-time MI processing methodology was found through a comparative study. The study shows that the novel method which combines Hjorth parameters and Welch-PSD coefficients as features - provides high accuracy and generalisability across subjects, as well as low computational complexity that allows it to be an optimal approach for real-time processing.

The real-time feasibility of the method was tested by navigating an object-retrieving robot in a maze, using three MI class signals. Fig. 1 outlines the flow 
for the real-time experiment conducted. The robot was able to successfully navigate the maze with high accuracy and low processing time. Object picking and retrieval were automated, thus reducing the number of modalities of control, alleviating user strain and reducing cost of resources. The intended application of this study was to aid the elderly and physically-handicapped in daily tasks.

The rest of the paper is organized as follows. Section 2 discusses relevant previous work done in the field, while section 3 introduces the datasets used for analysis. In section 4, various EEG-processing methodologies are compared on two-class data, and section 5 presents the results of the comparative study of two-classes. Section 6 discusses how the best methodology found was extended to three-class data and was used in a real-time robot navigation and object retrieval experiment. Section 7 presents the results of the experiment and section 8 provides a discussion of the findings. Concluding remarks are given in section 9.

\section{Literature survey}

A variety of EEG-based robots in the field of healthcare have been introduced in the past. An implementation of an obstacle-avoiding robot using artificial potential field (APF) and SSVEP (Steady state visually evoked potentials) has been proven to be successful [1]. However, the paper implemented continuous user control, causing strain to the user.

Another study, [2] performed wheelchair navigation and object picking with a robotic arm using shared-control. SSVEP was used for task selection and cervical movements were used to indicate navigational intent. This method alleviated user strain but could not be used for people with complete paralysis.

Considering the drawbacks of both approaches, this study implements a non-continuous shared-control approach. Obstacle avoidance is made autonomous to reduce user-strain, and only MI signals (unimodal) are considered for navigation.

The proposed work in [3] deeply studied the incidence of event-related desynchronization and synchronization (ERD and ERS) with MI signals and used it to achieve fast multi-class classification. The distinctive changes in spectral power were captured in real-time and used to control a BCI in [4]. Considering that MI signals show promise in real-time applications (fast classification), and given that the control is more intuitive, MI-based navigation was implemented in this work.

Extensive work has been done in the past in order to extract features that best represent MI signals. The work in [5] proposed the use of dual-tree complex wavelet transform (DTCWT) for signal decomposition as it overcame the problems of discrete wavelet transform (DWT) such as aliasing and power losses at the transaction bands, and the work in [6] compared several methods like wavelet packet decomposition (WPD), empirical mode decomposition (EMD), and DWT, and concluded that WPD was the superior method because it decomposed both the low and high frequency bands, creating a fine separation of relevant frequency bands. The study in [7] explored another implementation of WPD where features were extracted using a filter based on the Fisher criterion.

First-order and higher-order statistics such as skewness and kurtosis were studied in [6], and skewness and kurtosis were shown to provide a more efficient analysis of non-linear, non-stationary signals like EEG. Studies in $[8,9]$ presented the estimation of power spectral density (PSD) using the Welch method (Welch-PSD). The work in $[10,11,12]$ stated that PSD is a good feature set for three class problems due to the high signal to noise ratio. Papers $[13,14]$ implemented Hjorth parameters on frequency-filtered and subsequently time-windowed signals, while [15] employed them on wavelet decomposed signals. Hjorth features are derived from the variance parameter in the time-domain and are thus computationally inexpensive.

The aforementioned feature extraction methods that have shown promise for MI signals have been compared in this study. The aim was to find the most computationally efficient and accurate real-time feature extraction technique for MI signals, with the end-goal of robot navigation.

Classifiers like support vector machine (SVM) have been extensively used in BCI research [9] owing to their fast classification and good generalization capability. Paper [16] suggested the use of another commonly used classifier- logistic regression, since it does not require a Gaussian distribution of independent variables, thereby aiding the classification of EEG signals. These two classification methods were implemented in this study.

\section{Datasets}

This study employed the publicly available BCI Competition III datasets - Dataset IVa, and Dataset $\mathrm{V}$ - elaborated on in [17]. They have been outlined in Table 1. 
Table 1. Summary of the datasets employed in this study

\begin{tabular}{|c|c|c|c|c|c|}
\hline Dataset & Sub & No. of training cues & No. of testing cues & Total no. of cues & Classes \\
\hline \multirow{5}{*}{$\begin{array}{l}\text { BCI Competition } \\
\text { III, Dataset IVa }\end{array}$} & $a a$ & 168 & 112 & \multirow{5}{*}{$\begin{array}{c}280 \\
(3.5 \text { s per cue })\end{array}$} & \multirow{5}{*}{$\begin{array}{l}\text { 'Right' } \\
\text { 'Foot' }\end{array}$} \\
\hline & $a l$ & 224 & 56 & & \\
\hline & $a v$ & 84 & 196 & & \\
\hline & aw & 56 & 224 & & \\
\hline & ay & 28 & 252 & & \\
\hline \multirow{3}{*}{$\begin{array}{l}\text { BCI Competition } \\
\text { III, Dataset V }\end{array}$} & 1 & 48 & 16 & \multirow{3}{*}{$\begin{array}{c}63 \\
\text { (15 s per cue })\end{array}$} & \multirow{3}{*}{$\begin{array}{l}\text { 'Left' } \\
\text { 'Right' } \\
\text { 'Word' }\end{array}$} \\
\hline & 2 & 48 & 16 & & \\
\hline & 3 & 48 & 16 & & \\
\hline
\end{tabular}

Table 2. Windowing of the three-class dataset

\begin{tabular}{|c|c|c|}
\hline Sub & $\begin{array}{c}\text { No. of Training } \\
\text { Windows }\end{array}$ & $\begin{array}{c}\text { No. of Testing } \\
\text { Windows }\end{array}$ \\
\hline $\boldsymbol{1}$ & 716 & 236 \\
\hline $\mathbf{2}$ & 711 & 238 \\
\hline $\mathbf{3}$ & 714 & 239 \\
\hline
\end{tabular}

Dataset IVa comprised of five subjects where the number of training cues varied per subject. Each cue was $3.5 \mathrm{~s}$ long and belonged to one of the two-classes 'right' and 'foot'. As provided in the competition, the signals associated with labelled cues were used as training data, and those associated with unlabelled cues were used as testing data. Data from 118 electrodes was collected. The dataset provides a good baseline estimate of the performance of the proposed methodology because it contains less data, as would commercial EEG-based applications. The comparative study detailed in this paper was done using this dataset.

Dataset V comprised of data of three subjects that was recorded using 32 electrodes. Each subject's data consisted of 48 training cues and 16 testing cues, belonging to the three classes 'left', 'right', and 'word'. Each cue was $15 \mathrm{~s}$ long and was broken down into non-overlapping windows of $1 \mathrm{~s}$ with the corresponding class label given to each window, keeping in mind real-time application (fast execution). Processing was done on each $1 \mathrm{~s}$ window rather than on the entire cue signal. Windows corresponding to the labelled cues were used as training data, while the rest were used as testing data. The break-up of windows for each subject are given in Table 2 .

In this study, Dataset $V$ was used for the real-time robot navigational experiment. The three classes corresponded to the navigational commands 'Turn Left', 'Turn Right' and 'Go Forward' respectively.

\section{Methodology}

A variety of methods have been introduced over the years to extract features from EEG signals. A few such methods, as seen in Section 2, were implemented on two-class data (Dataset IVa) and compared in this study.

\subsection{Pre-processing}

Eight electrode channels namely, ' $\mathrm{CP} 1$ ', 'CP2', 'C3', 'Cz', 'C4', 'P3', 'Pz', and 'P4' of the international 10/20 system, were selected based on the type of MI action being captured [18].

The eight channels of data were band pass filtered over 8-35 Hz using a second order Butterworth bandpass filter to remove high frequency noise, $\mathrm{AC}$ line noise, and DC content. This was performed prior to feature extraction, with the exception of Hjorth feature extraction where the bandpass frequency range was a parameter to be found for each subject individually.

\subsection{Feature extraction}

\subsubsection{Method 1: DTCWT}

The first feature extraction method that was implemented used the wavelet-based signal decomposition technique DTCWT. This technique is based on a common orthogonal-wavelet-based decomposition method known as DWT. It is further improved to overcome DWT's aliasing and power-loss issues [5]. To implement DTCWT, two DWTs are operated in parallel to calculate the real and imaginary parts of the transform separately. The resulting detail and approximation coefficients at each level are computed by adding the corresponding real and imaginary coefficients together. In this study, the signals from the eight channels were decomposed to four levels using the near-symmetric wavelet for level 1 , and the qshift wavelet for the remaining levels. The first-order 
statistics mean, standard deviation, energy, rootmean-square (RMS) value, and the higher-order statistics skewness and kurtosis were found only on the subbands D2, D3, and D4 which corresponded to the detail coefficient of frequencies 14.75-16.43, 11.3813.06, and 9.69-11.38 Hz respectively, thus extracting features corresponding to the $\mathrm{Mu}$ and Beta rhythms of MI signals [6]. This resulted in a 144length feature vector per cue.

\subsubsection{Method 2: WPD}

Another wavelet-based decomposition technique is WPD. An extension of DWT, WPD offers better frequency resolution as it produces more discriminating features than DWT. This is due to the fact that unlike DWT which only decomposes the approximation coefficients at each level, WPD decomposes both the approximation and detail coefficients, producing a binary tree. The approximation signal at level $j\left(W_{j 0}\right)$ Eq. (1) and the detail signal at the same level $\left(W_{j 1}\right)$ Eq. (2) is given as follows:

$$
\begin{aligned}
& W_{\mathrm{j} 0}[i]=\sum_{k} \mathrm{x}[\mathrm{k}] \mathrm{l}[2 \mathrm{i}-\mathrm{k}] \\
& W_{\mathrm{j} 1}[i]=\sum_{k} \mathrm{x}[\mathrm{k}] \mathrm{l}[2 \mathrm{i}-\mathrm{k}]
\end{aligned}
$$

where $x$ is the input signal and $h$ and $l$ are the high and low pass filters respectively.

WPD was implemented up to four levels, giving sixteen subbands with equally divided frequency ranges between $8-35 \mathrm{~Hz}$ per channel. The Daubechies wavelet of the order four was used and mean, variance, RMS, skewness, and kurtosis features were found for all the subband coefficients resulting in a 640-length feature vector per cue.

\subsubsection{Method 3: Welch-PSD}

In this method, features were obtained by finding power spectral density using Welch's method [10]. It is a form of non-parametric computation that finds the weighted sum of the periodograms of overlapping windows of the signal. Welch-PSD is calculated as follows:

$$
\hat{I}_{x x}^{W}[\omega]=\frac{1}{P} \sum_{p=0}^{P-1} \hat{I}_{x x}^{(p)}(\omega)
$$

where the number of segments is denoted by $\mathrm{P}$ and $\hat{I}_{x x}^{(p)}(\omega)$ is the periodogram per windowed segment, the average of which is denoted by $\hat{I}_{x x}^{W}(\omega)[19]$.
A Hanning window function was used and $0.5 \mathrm{~s}$ windows were extracted from the channels with a $50 \%$ overlap. This resulted in 26 central frequencies ranging from 0 to $50 \mathrm{~Hz}$. The Welch-PSD coefficients obtained after computation were used as features. This resulted in a 208-length feature vector per cue.

\subsubsection{Method 4: Hjorth parameters}

Hjorth parameters extract statistical properties of EEG signals in the time-domain. They consist of three types - activity (Act), mobility (Mob), and complexity (Com). Activity describes the signal power, mobility determines average frequency, and complexity shows variation in frequency [20]. These are computed as shown in Eq. (4), Eq. (5) and Eq. (6) respectively.

$$
\begin{aligned}
& A c t=\operatorname{variance}(y(t)) \\
& M o b=\sqrt{\frac{\operatorname{Act}(y \prime(t))}{\operatorname{Act}(y(t))}} \\
& \text { Com }=\frac{\operatorname{Mob}(y \prime(t))}{\operatorname{Mob}(y(t))}
\end{aligned}
$$

Here, $y(t)$ is the input signal, which in this case is the data from the electrode channels, and $y^{\prime}(t)$ is its derivative.

Twenty-one second order Butterworth bandpass filters consisting of all combinations of 5, 8, 13, 15, 20,25 , and $30 \mathrm{~Hz}$ were applied to the signal prior to finding the Hjorth parameters. A window size of $1 \mathrm{~s}$ was used with a $0.5 \mathrm{~s}$ overlap for each cue. The best combination of frequency band, Hjorth parameter, and window per cue was found using the Fisher ratio [13]. The best combination for each subject resulted in an 8-length feature vector per cue which was then used for the final classification.

\subsubsection{Method 5: Hjorth parameters and WPD}

This method combines WPD with Hjorth parameters. The signals were decomposed using WPD to four levels (16 subbands with equally divided frequency ranges between $8-35 \mathrm{~Hz}$ per channel) and reconstructed with the best four subbands which varied per subject and was found using five-fold cross-validation. Features were then extracted from the reconstructed signals - skewness, kurtosis, and Hjorth parameters. Considering that the 
Table 3. Two-class comparative study results

\begin{tabular}{|c|l|c|c|c|c|c|c|}
\hline \multirow{2}{*}{$\begin{array}{c}\text { Sl. } \\
\text { No. }\end{array}$} & \multicolumn{1}{|c|}{ Methods } & \multicolumn{4}{|c|}{ Subject } & \multirow{2}{*}{ Average } \\
\cline { 3 - 7 } & & $\boldsymbol{a} \boldsymbol{a}$ & $\boldsymbol{a l}$ & $\boldsymbol{a} \boldsymbol{v}$ & $\boldsymbol{a} \boldsymbol{w}$ & $\boldsymbol{a} \boldsymbol{y}$ & \\
\hline $\mathbf{1 .}$ & DTCWT & 0.70 & 0.91 & 0.58 & 0.77 & 0.69 & 0.73 \\
\hline $\mathbf{2 .}$ & WPD & 0.68 & 0.84 & 0.57 & 0.71 & 0.59 & 0.68 \\
\hline 3. & Welch PSD & 0.54 & 0.75 & 0.59 & 0.67 & 0.48 & 0.61 \\
\hline 4. & Hjorth & 0.69 & 0.91 & 0.62 & 0.84 & 0.85 & 0.78 \\
\hline 5. & Hjorth-WPD & 0.75 & 0.95 & 0.62 & 0.88 & 0.70 & 0.78 \\
\hline $\mathbf{6 .}$ & Hjorth-Welch PSD & $\mathbf{0 . 7 8}$ & $\mathbf{0 . 9 8}$ & $\mathbf{0 . 7 0}$ & $\mathbf{0 . 9 0}$ & $\mathbf{0 . 8 4}$ & $\mathbf{0 . 8 4}$ \\
\hline
\end{tabular}

dominant frequency band varies per subject, extracting the best frequency bands with a fine separation of frequency allowed for specific subject dependent features to be extracted.WPD resulted in a large feature vector of length 640. Performing subband selection reduced the feature vector length to 40 . This step is crucial for small datasets as it reduces overfitting.

\subsubsection{Method 6: Hjorth parameters and Welch-PSD}

Here, Hjorth parameters and Welch-PSD coefficients were used as features. Welch PSD was computed on the cue signal and the hyperparameters window size and overlap - were found using fivefold cross-validation resulting in different feature vector lengths. This gave subject-dependent frequency resolutions. Unlike in method 4, Hjorth parameters in this method were found on the entire cue. The feature vector length ranged from 48-112 across the five subjects. An $8-35 \mathrm{~Hz}$ second order Butterworth bandpass filter was used prior to feature extraction.

\section{Results of the comparative study}

For all methods, with the exception of method 4, Select-k-Best, a correlation-based feature selector was chosen for feature selection. The number of features chosen was found using a grid search with fivefold cross-validation. The performance of the methods was evaluated on the two-class data using a binary SVM classifier with a linear kernel.

The results of the feature extraction comparative study are shown in Table 3. As seen in the table, the first five feature extraction methods that were attempted resulted in average accuracies of less than 0.80 across all five subjects. The novel combination of Hjorth parameters and Welch-PSD, proposed in this work, gave the highest average accuracy of 0.84 over all the subjects, with the highest accuracy of 0.98 for 'al'. This can be due to the subject dependent frequency resolution that the proposed implementation of Welch-PSD provides, combined with the highly informative time-domain features offered by the Hjorth parameters. The subject-dependent window size and overlap used made the method more generalizable across subjects as compared to previously attempted methods as discussed in Section 8.

Moreover, this combination yielded the lowest model training time of $5.35 \mathrm{~s}$ for two-class data, as seen in Fig. 2. Training time showed a very gradual linear increase with the increase in the number of training cues, making the feature extraction technique suitable for real-time applications. This can be accounted for by the low computational complexity. Six $N$-point Fast Fourier Transforms (FFT) (complexity of $O\left(N \log _{2} N\right)$ [21]) are needed for WelchPSD to be performed on each cue, and additionally, a variance-based Hjorth feature extraction must be performed on each cue. This is comparable with DTCWT $(2 * O(N))$, but is far better than WPD $\left(O\left(L * N \log _{2} N\right)\right)$. Where $N$ is the length of the signal for all the methods mentioned, and $L$ is the number of decomposition levels for WPD.

\section{Real-time robot navigation}

In order to provide three navigational commands to the robot, the novel methodology - method 6 found in the comparative study was employed on the three-class dataset (Dataset V).

The dataset was windowed as mentioned in Section 3 and processed using the Hjorth parameters and Welch-PSD method described in Section 4. The same eight electrode channels used for two-class processing were chosen for three-class processing. The added class, 'word', represented the task of mentally forming words that begin with the same given letter. This task comes under the domain of speech processing and primarily uses the parietal lobe of the brain [22]. The 'P3', P4', and 'Pz' 
Table 4. Results of three-class processing

\begin{tabular}{|c|c|c|c|c|}
\hline Sub & Accuracy & F1 score & Precision & Recall \\
\hline $\boldsymbol{1}$ & $\mathbf{0 . 7 8}$ & 0.78 & 0.78 & 0.78 \\
\hline $\boldsymbol{2}$ & $\mathbf{0 . 6 3}$ & 0.63 & 0.63 & 0.63 \\
\hline $\boldsymbol{3}$ & $\mathbf{0 . 5 8}$ & 0.57 & 0.58 & 0.58 \\
\hline Avg. & $\mathbf{0 . 6 6}$ & 0.66 & 0.66 & 0.66 \\
\hline
\end{tabular}

channels used for two-class processing falls in the parietal region and therefore no extra channels were needed. Logistic regression (one-vs-rest) performed better than SVM for three-class classification and was therefore used as the classifier for this dataset.

The performance of the three-class model is shown in Table 4. An average accuracy of 0.66 across all subjects was achieved with subject 1 performing the best. The class 'word' had the highest average F1 score of 0.73 followed by the classes 'right' and 'left' with 0.65 and 0.57 respectively. The proposed method maintained a low training time of $12.34 \mathrm{~s}$ for three-class data.

Since subject 1's data showed the highest accuracy of 0.78 , it was used as the final input data for the robot navigational experiment.

The experiment was performed to verify the feasibility of the proposed method in a real-time application. A simple object-retrieving robot prototype was built with the intention of aiding the elderly and individuals with motor disabilities with their daily tasks. The robot was built using the Raspberry Pi $3 \mathrm{~B}+$, which processed the input MI signals using the novel method and sent all control signals to the robot peripherals. Three ultrasonic sensors placed on the front, right, and left sides of the robot helped in obstacle avoidance.

An arm with two degrees of freedom was controlled using two servo motors (torque - 9.4

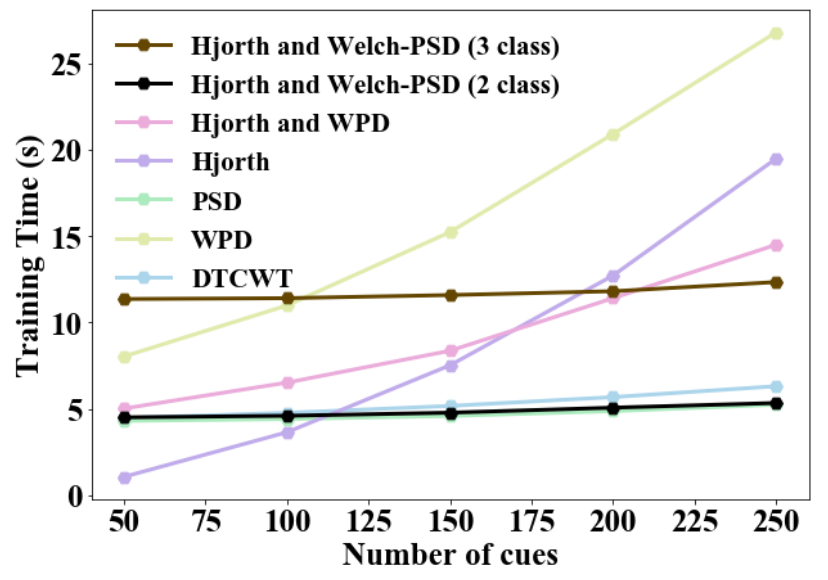

Figure. 2 Training time (s) vs. number of cues

$\mathrm{kg} / \mathrm{cm}$ ), and was used to pick objects. The gripping action was controlled with another servo motor (torque $-1.3 \mathrm{~kg} / \mathrm{cm}$ ). A monocular camera was used for object detection and the feed was transmitted wirelessly (via TCP) to a remote computer system. This would give the user a good view of the path that the robot was taking and would enable them to provide a navigational command when required. The Raspberry Pi was powered by a power bank, and two rechargeable batteries powered the two DC motors (300 RPM) that moved the robot. The total build was cost-effective.

A maze was built as per the specifications shown in Fig. 3 (a). As discussed in previous sections, channel selection and $1 \mathrm{~s}$ windowing were performed on subject 1's data, and the training-data was processed using the novel methodology and used for model training. The robot - now operating on the trained model - was placed at one entrance of the maze. Aided by the three ultrasonic sensors, it moved through the maze until an intersection was reached. Upon reaching the intersection, the robot waited for a navigational command.

Three random testing-data windows of the class

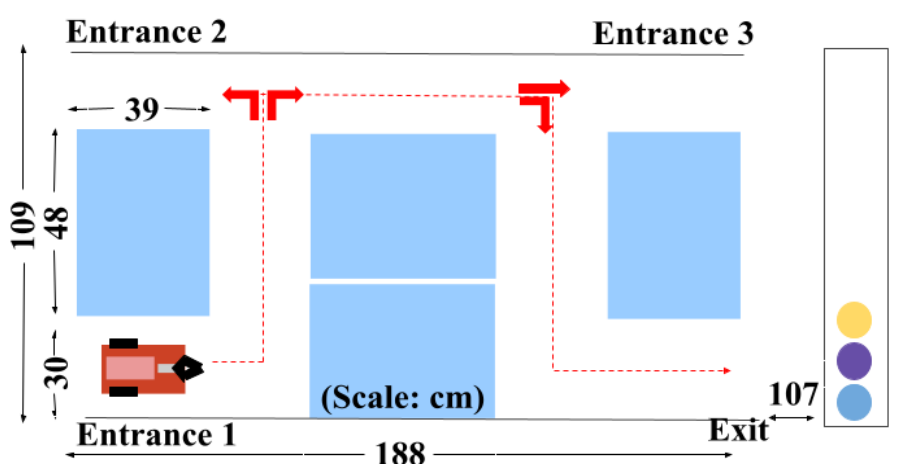

(a)

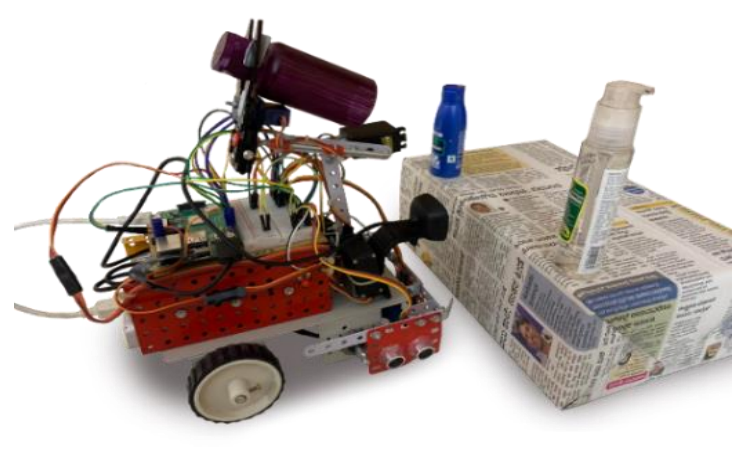

(b)

Figure. 3: (a) Maze specifications and (b) Robot picking up the desired object 
Table 5. Results of robot navigation experiment

\begin{tabular}{|c|c|c|c|c|}
\hline Entrance & $\begin{array}{c}\text { Path Length and } \\
\text { Number of Turns }\end{array}$ & $\begin{array}{c}\text { Average Maze Navi- } \\
\text { gation Time (s) }\end{array}$ & $\begin{array}{c}\text { Average Maze Retrac- } \\
\text { ing Time (s) }\end{array}$ & $\begin{array}{c}\text { Distance (cm) / Av- } \\
\text { erage Time (s) }\end{array}$ \\
\hline $\mathbf{1}$ & $422 \mathrm{~cm}(4$ turns) & 61.71 & 60.48 & 6.94 \\
\hline $\mathbf{2}$ & $363 \mathrm{~cm}(2$ turns) & 40.11 & 45.92 & 8.47 \\
\hline $\mathbf{3}$ & $300 \mathrm{~cm}(2$ turns) & 38.41 & 38.09 & 7.87 \\
\hline Avg. & $\mathbf{3 6 2}$ cm (3 turns) & $\mathbf{1 4 0 . 2 3}$ & $\mathbf{4 8 . 1 6}$ & $\mathbf{7 . 7 6}$ \\
\hline
\end{tabular}

corresponding to the intended command was inputted to the robot - 'right', 'left', and 'word' were used for the commands Turn Right, Turn Left, and Go Forward respectively. The signal windows were processed and classified using the proposed method, with no knowledge of the class label. The final navigational command sent to the robot corresponded to the majority of the three consecutive classifications. The process of finding the command by using three consecutive random windows is termed as making one 'decision'.

After receiving the command, the robot crosschecked if the path corresponding to the given command was obstructed or not. If the path was obstructed - as determined by the ultrasonic sensors the robot assumed misclassification and repeated the process by taking three more inputs, thus improving the navigational performance. However, if the path was unobstructed but the navigational command did not match the intended direction of motion, the message 'failure' was flashed on the computer system, indicating that the robot failed the navigation process.

Once the robot reached the end of the maze, it detected and automatically picked-up the desired object - that was predefined based on its colour - out of a set of objects. (Fig. 3 (b)). The robot then retrieved the object using automatic path-retracing. This shared-control strategy allows high-level actions navigational decisions - to be controlled by the user, leaving the low-level control to the robot. Further, since the input signal would be required only at a decision-making point, the user would feel considerably less mental strain than they would in the continuous user-control approach.

\section{Results of robot navigation}

The real-time simulated navigation experiment was conducted as described in the previous section, and the robot was successful in its navigation nine out of ten times via all three circuits. It moved at an average speed of $7.76 \mathrm{~cm}$ per $\mathrm{s}$ and the average time taken to complete each circuit is given in Table 5 .

The average maze retracing time included the few seconds taken for the robot to re-enter the maze after picking up the object. Navigation time and retracing time were comparable, implying that the speed of EEG processing and classification was high. This verified the utility of the novel method for realtime applications.

The time taken to traverse the longest circuit, retrieve the object, and retrace back to the starting point was an average of $2 \mathrm{~min} 28 \mathrm{~s}$. The timing overheads included a one-time model training time of only $17.34 \mathrm{~s}$ and an object-picking time of $27.23 \mathrm{~s}$. Training is needed to be done only once per user.

Overall, the robot navigated the maze with good accuracy, advantageously short training time, and low computational power. The strength of the novel method was further validated by considering hundred navigational 'decisions' per class of signals. While the average accuracy for subject 1 was only 0.78 , the average accuracy of hundred decisions was 0.82 , as seen in Table 6 . Thus, the use of three short signal windows (1s each) to make each navigational decision resulted in a higher probability that the robot reached its destination.

\section{Discussion}

This section discusses the strength of the proposed method (found through the comparative study) in the context of recent existing studies that have used Datasets IVa and V from BCI Competition III.

The work in [23, 24] implemented common spatial patterns (CSP) and spatially regularized CSP using eighteen electrode channels, yielding average accuracies of 0.80 and 0.79 respectively on the twoclass Dataset IVa. The work in [25] once again used eighteen channels on the two-class data and an extensive method combining CSP with attractor metagene (AM) and the Bat optimization algorithm of SVM to select the most discriminatory CSP features resulting in an accuracy of 0.85 . The work in [26] employed a clustering-technique based least-square SVM and yielded an average accuracy of 0.84 using all 118 channels.

The proposed novel method in this study provides an average accuracy of 0.84 across all subjects. This proved to be better than the accuracies 
Table 6. Navigational decision accuracy

\begin{tabular}{|c|c|c|c|c|}
\hline \multirow{2}{*}{ Sub } & \multicolumn{2}{|c|}{$\begin{array}{c}\text { No. of Correct Navigational } \\
\text { Decisions (Hundred Trials) }\end{array}$} & \multirow{2}{*}{$\begin{array}{c}\text { Average } \\
\text { Decision } \\
\text { Accuracy }\end{array}$} \\
\cline { 2 - 5 } & $\begin{array}{c}\text { Turn } \\
\text { Right }\end{array}$ & $\begin{array}{c}\text { Turn } \\
\text { Left }\end{array}$ & $\begin{array}{c}\text { Go For- } \\
\text { ward }\end{array}$ & (1) \\
\hline $\boldsymbol{1}$ & 77 & 85 & 84 & $\mathbf{0 . 8 2}$ \\
\hline $\mathbf{2}$ & 51 & 59 & 80 & $\mathbf{0 . 6 3}$ \\
\hline $\mathbf{3}$ & 43 & 70 & 62 & $\mathbf{0 . 5 8}$ \\
\hline
\end{tabular}

achieved in papers [23, 24], and only less than that of paper [25] by 0.01 . These three studies employed eighteen electrode channels, while the proposed method used only eight channels. The work in [26] utilized all 118 channels available in Dataset IVa, but only achieved similar accuracies as the proposed method which used a considerably less number of channels.

Moreover, the proposed method showed more consistent accuracies across subjects as compared to the other studies, thereby further proving generalizability. Additionally, the proposed method was advantageous in its lower computational complexity and processing time (5.35s for two-class data), proving to be more useful for real-time applications.

The winners of the competition for the three-class Dataset V [17] achieved an average accuracy of 0.69 by working with the pre-computed PSD feature set that was optionally provided by the competition. The team that came first using the raw-data employed multi-class CSP on fifteen channels and achieved an accuracy of 0.63 [17]. The paper [27] implemented separable common spatio-spectral pattern on the rawdata and achieved an accuracy of 0.65 . The work in [28] employed discrete Fourier transform (DFT) and hidden Markov models (HMM) on 2s windows of raw-data from all 32 electrode channels and achieved a higher accuracy of 0.70 .

The proposed method in this study, however, used the raw-data and achieved an average accuracy of 0.66 - a higher accuracy than that of the raw-data methods studied in $[17,27]$. Although the work in [28] yielded a higher accuracy, the proposed method in this study used only eight channels and shorter $1 \mathrm{~s}$ windows.

Overall, it can be seen that the novel method introduced in this study works well on both two-class and three-class MI data and is computationally less complex, making it highly suitable for real-time applications.

\section{Conclusion}

In this study, conventional feature extraction techniques were examined and compared on the BCI Competition III Dataset IVa (two-classes). A novel combination of Welch PSD and Hjorth parameters was found to provide the best accuracy of 0.84 across five subjects, with two subjects crossing 0.90 .

The approach uniquely combined time-domain analysis using Hjorth parameters, with frequency-domain analysis using Welch PSD, allowing for low computational complexity, good generalizability across subjects and short training time $(12.34 \mathrm{~s}$ for three-class data). Accuracies were comparable with other studies that utilized the same datasets.

The method, when applied on Dataset V (threeclasses), gave an average accuracy of 0.66 across three subjects. Using subject 1's data from this dataset, a robot navigation experiment using three motor commands - 'Turn Right', 'Turn Left', and 'Go Forward' - was performed. The robot successfully navigated all three circuits of the maze with good speed, thus verifying the feasibility of the novel method for real-time applications.

The use of three consecutive, $1 \mathrm{~s}$ long signal windows to make each navigational decision improved the navigational performance, as did the misclassification detection using the ultrasonic sensors. User-fatigue caused by prolonged mental visualization is alleviated by the shared-control framework employed. Signals are read only in short bursts only when a navigational decision is to be made, and object-retrieval and path-retracing is made autonomous.

In conclusion, the novel method and shared-control framework proposed in this study can prove to be beneficial in real time applications. Particularly, daily needs of the elderly and the physically handicapped in home and hospital environments can be met. The cost-effective build of the robot allows for commercialization of the device, and the small number of electrode channels required makes it compatible with cheap and portable EEG signal acquisition equipment.

Future work will primarily focus on acquiring MI signals in real-time. The proposed methodology must be implemented on more subjects Additionally, monocular simultaneous localization and mapping (SLAM) will be implemented on a multi-terrain quadruped to enable navigation in a real home environment. Finally, the quadruped will be 3D printed to further reduce cost.

\section{Conflicts of Interest}

The authors declare no conflict of interest. 


\section{Author Contributions}

Conceptualization, visualization: Dr. Niranjana Krupa, M. J. Niegil Francis, Mihika Preethi Keran, and Rachana Chetan; Methodology, software, validation, formal analysis, investigation, writing - original draft preparation: M. J. Niegil Francis, Mihika Preethi Keran, and Rachana Chetan; Supervision, project administration, writing - review and editing: Dr. Niranjana Krupa; Funding acquisition, resources: PES University

\section{Acknowledgments}

This work was supported by the Department of Electronics and Communication, PES University, Bangalore, India.

\section{References}

[1] Y. Liu, Z. Li, T. Zhang, and S. Zhao, "Brain-Robot Interface-Based Navigation Control of a Mobile Robot in Corridor Environments", IEEE Transactions on Systems, Man, and Cybernetics: Systems, Vol. 50, No. 8, pp. 3047-3058, 2020.

[2] F. Achic, J. Montero, C. Penaloza, and F. Cuellar, "Hybrid BCI system to operate an electric wheelchair and a robotic arm for navigation and manipulation tasks", In: 2016 IEEE Workshop on Advanced Robotics and its Social Impacts (ARSO), Shanghai, China, pp. 249-254, 2016.

[3] S. Seifzadeh, M. Rezaei, K. Faez, and M. Amiri, "Fast and Efficient Four-class Motor Imagery Electroencephalography Signal Analysis Using Common Spatial Pattern- Ridge Regression Algorithm for the Purpose of Brain-Computer Interface", Journal of Medical Signals and Sensors, Vol. 7, No. 2, pp. 80-85, 2017.

[4] N. Brodu, F. Lotte, and A. Lecuyer, "Comparative Study of Band Power Extraction Techniques for Motor Imagery Classification", In: Proc. of IEEE Symposium on Computational Intelligence, Cognitive Algorithms, Mind and Brain, Paris, France, pp. 1-6, 2011.

[5] N. S. Malan and S. Sharma, "Feature Selection Using Regularized Neighbourhood Component Analysis to Enhance the Classification Performance of Motor Imagery Signals", Computers in Biology and Medicine, Vol. 107, pp. 118-126, 2019.

[6] J. Kevric and A. Subasi, "Comparison of Signal Decomposition Methods in Classification of EEG Signals for Motor-Imagery BCI System", Biomedical Signal Processing and Control, Vol. 31, pp. 398-406, 2017.
[7] W. Ting, Y. Guo-Zheng, Y. Bang-hua, and S. Hong, "EEG Feature Extraction Based on Wavelet Packet Decomposition for Brain Computer Interface", Measurement, Vol. 41, No. 6, pp. 618-625, 2008.

[8] M. A. Naderi and H. M. Nasab, "Analysis and Classification of EEG Signals using Spectral Analysis and Recurrent Neural Networks", In: Proc. of Iranian Conf. of Biomedical Engineering, Isfahan, Iran, pp. 1-4, 2010.

[9] H. Pawel, G. Prasad, T. M. McGinnity, and D. H. Coyle, "Comparative Analysis of Spectral Approaches to Feature Extraction for EEGbased Motor Imagery Classification", IEEE Transactions on Neural Systems and Rehabilitation Engineering, Vol. 16, No. 4, pp. 317-326, 2008.

[10] O. M. Solomon, PSD Computations Using Welch's Method, Sandia National Laboratories, Albuquerque, USA, 1991.

[11] S. N. Resalat and V. Saba, "A Study of Various Feature Extraction Methods on a Motor Imagery-based Brain Computer Interface System", Basic and Clinical Neuroscience, Vol. 7, No. 1, pp. 13-19, 2016.

[12] F. Mohamed, S. F. Ahmed, Z. Ibrahim, and S. Yaacob, "Comparison of Features Based on Spectral Estimation for the Analysis of EEG Signals in Driver Behavior", In: Proc. of International Conf. on Computational Approach in Smart Systems Design and Application, Kuching, Malaysia, pp. 1-7, 2018.

[13] S. H. Oh, Y. R. Lee, and H. N. Kim, "A Novel EEG Feature Extraction Method Using Hjorth Parameter", International Journal of Electronics and Electrical Engineering, Vol. 2, No. 2, pp. 106-110, 2014.

[14] B. Hjorth, "EEG Analysis Based on Time Domain Properties", Electroencephalography and Clinical Neurophysiology, Vol. 29, No. 3, pp. 306-310, 1970.

[15] M. Mumtaz, M. Afzal, and A. Mushtaq, "Sensorimotor Cortex EEG Signal Classification Using Hidden Markov Models and Wavelet Decomposition", In: Proc. of International Symposium on Signal Processing and Information Technology, Louisville, USA, pp. 375-381, 2018.

[16] C. F. C. Soeiro, "EEG Signal Analysis Using PCA and Logistic Regression", In: Proc. of XXVI Brazilian Congress on Biomedical Engineering, Reo de Janerio, Brazil, pp. 175-180, 2019.

[17] B. Blankertz, K. R. Muller, and D. J. Krusienski, "The BCI competition III: Validating Alternative Approaches to Actual BCI Problems", IEEE 
Transactions on Neural Systems and Rehabilitation Engineering, Vol. 14, No. 2, pp. 153-159, 2006.

[18] J. Abougharbia, O. Attallah, M. Tamazin, and A. A. A. Nasser, "A Novel BCI System Based on Hybrid Features for Classifying Motor Imagery Tasks", In: Proc. of International Conf. on Image Processing Theory, Tools and Applications, Istanbul, Turkey, pp. 1-6, 2019.

[19] M. D. Basar, A. D. Duru, and A. Akan, "Emotional state detection based on common spatial patterns of EEG", Signal, Image and Video Processing, Vol. 14, No. 3, pp.473-481, 2020.

[20] A. Patil, C. Deshmukh, and A. R. Panat, "Feature extraction of EEG for emotion recognition using Hjorth features and higher order crossings", In: Proc. of 2016 Conf. on Advances in Signal Processing, Pune, India, pp. 429-434, 2016.

[21] K. K. Parhi and M. Ayinala, "Low-Complexity Welch Power Spectral Density Computation", IEEE Transactions on Circuits and Systems I: Regular Papers, Vol. 61, No. 1, pp. 172-182, 2014.

[22] H. B. Coslett, and M. F. Schwartz, Handbook of Clinical Neurology, Vol. 151, Elsevier, Amsterdam, Netherlands, 2018.

[23] S. Selim, M. Tantawi, H. Shedeed, and A. Badr, "A Comparative Analysis of Different Feature Extraction Techniques for Motor Imagery Based BCI System”, In: Proc. of the International Conf. on Artificial Intelligence and Computer Vision, Cairo, Egypt, pp. 740-749, 2020.

[24] F. Lotte and C. Guan, "Regularizing Common Spatial Patterns to Improve BCI Designs: Unified Theory and New Algorithms", IEEE Transactions on Biomedical Engineering, Vol. 58, No. 2, pp. 355-362, 2011.

[25] S. Selim, M. M. Tantawi, H. A. Shedeed, and A. Badr, "A CSP $\backslash A M-B A-S V M$ Approach for Motor Imagery BCI System", IEEE Access, Vol. 6, pp. 49192-49208, 2018.

[26] Siuly, Y. Li, and PP. Wen, "Clustering technique-based least square support vector machine for EEG signal classification", Computer Methods and Programs in Biomedicine, Vol. 104, No. 3, pp. 358-372, 2011.

[27] J. Petersen, H. K. Iversen, and S. Puthusserypady, "Motor Imagery based Brain Computer Interface Paradigm for Upper Limb Stroke Rehabilitation", In: Proc. of 40th Annual International Conf. of the IEEE Engineering in Medicine and Biology Society, Honolulu, Hawaii, pp. 1960-1963, 2018.
[28] S. Nasehi and H. Pourghassem, "Mental Task Classification Based on HMM and BPNN", In: Proc. of International Conf. on Communication Systems and Network Technologies, Gwalior, India, pp. 210-214, 2013. 